Check for updates

Cite this: RSC Adv., 2017, 7, 27431

Received 14th February 2017 Accepted 14th April 2017

DOI: $10.1039 / \mathrm{c} 7 \mathrm{ra01820 \textrm {g }}$

rsc.li/rsc-advances

\section{Liposome encapsulation attenuated venenum bufonis induced vascular irritation in rabbit ear vein via regulating TLR/MAPK/NF- $\kappa$ B pathway ${ }^{\dagger}$}

\author{
Qin Cao, ${ }^{\text {ab }}$ Jianming Guo, ${ }^{\mathrm{b}}$ Dawei Qian, ${ }^{\mathrm{b}}$ Hongyue Ma, ${ }^{\mathrm{b}}$ Yunru Peng, ${ }^{\mathrm{c}}$ Erxin Shang, ${ }^{\mathrm{b}}$ \\ Buchang Zhao ${ }^{d}$ and Jinao Duan (D)*ab
}

Delisheng injection (DLS), composed of venenum bufonis (VB), Chinese blister beetle (CB), red ginseng (RG) and Astragalus membranaceus (AM), is a Chinese medicine injection with good efficacy for the treatment of different types of cancers, while the side effects of DLS, such as vascular irritation and inflammation, restrict its wide clinical applications. Based on our previous study, VB is a main contributor for the vascular irritation of DLS. To reveal the possible mechanism involved in VB-induced vascular irritation is of practical importance. Moreover, to find a way to alleviate VB-induced vascular irritation is of great necessity. In our present study, venenum bufonis liposome (VBL) was prepared by thin film dispersion method to attenuate VB-induced vascular irritation. Vascular irritation of VB and its liposome were evaluated in a rabbit model. Rabbits were divided into three groups: (1) the control group (K); (2) the VB group (VB); (3) the VBL group (VBL). Animals administrated with VB or VBL $0.12 \mathrm{mg} \mathrm{kg}^{-1}$ were sacrificed at $6 \mathrm{~h}$. Inflammatory cytokines and TLR/MAPK/NF- $K B$ pathway-related proteins were examined, respectively. Our results showed that VB

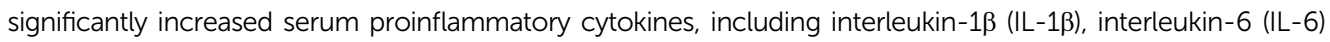
and tumor necrosis factor- $\alpha$ (TNF- $\alpha$ ), while it led to decreased anti-inflammatory cytokines interleukin-10 (IL-10) after intravenous administration. VB-induced vascular irritation was further confirmed by histopathological evaluation. Moreover, the results obtained from western blot and immunohistochemical analysis revealed that VB activated TLR/MAPK/NF- $\kappa B$ signaling pathway involving TLR2, TLR4, MyD88, $\mathrm{p}$-Erk, $p-J N K, p-P 38, p-1 \kappa B \alpha$ and $p-N F-\kappa B$ p65 protein. VB liposome decreased the VB-induced overexpression of TLR2, TLR4, MyD88, p-Erk, p-JNK, p-P38, $p-I_{\kappa} B \alpha$ and $p-N F-\kappa B$ p65. The anticancer activity of VB and VBL was also evaluated, and VBL still showed anticancer activity both in vivo and in vitro. Taken together, our finding demonstrated that VB induced vascular irritation was related to the regulation of the TLR/MAPK/NF$\kappa \mathrm{B}$ signaling pathway, and liposome encapsulation significantly attenuated VB-induced vascular irritation while maintaining its anticancer activity. The results of this study have important practical significance in guiding the safety clinical application of Chinese medicine injection.

\section{Introduction}

Delisheng injection (DLS), composed of venenum bufomis (VB), Chinese blister beetle (CB), red ginseng (RG) and Astragalus membranaceus (AM), is developed by modern preparation technology $y^{1}$ and demonstrates good efficacy for the treatment of hepatic carcinoma, ${ }^{2-4}$ lung cancer ${ }^{5}$ and some other types of

${ }^{a}$ College of Traditional Chinese Medicine, China Pharmaceutical University, Nanjing 210009, China. E-mail: dja@njutcm.edu.cn; Fax: +86-258-5811-116; Tel: +86-2585811-116

${ }^{b}$ Jiangsu Collaborative Innovation Center of Chinese Medicinal Resources Industrialization, Nanjing University of Chinese Medicine, Nanjing 210023, China

'Jiangsu Academy of Traditional Chinese Medicine, Nanjing 210028, China

${ }^{d}$ Buchang Pharma., Heze 274000, China

$\dagger$ Electronic supplementary information (ESI) available. See DOI: $10.1039 / \mathrm{c} 7 \mathrm{ra} 01820 \mathrm{~g}$ cancers. During its clinical practice, it was found that DLS caused varying degrees of vascular irritation, with the main symptoms to be red, swelling, pain beyond toleration at the site of injection and the surroundings. ${ }^{6-8}$ DLS induced vascular irritation not only affects its therapeutic effect, but also limits its wide range of clinical applications.

Based on macroscopic observation and histopathological results, our previous study showed that DLS, VB and CB exhibited different degrees of irritation on rabbit ear vein, and VB was the main contributor for the vascular irritation of DLS (ESI†). To reveal the possible mechanism involved in the vascular irritation is of practical guidance. Moreover, to find a way to alleviate VB-induced vascular irritation is of great necessity.

The application of liposomes for targeted drug delivery has been widely used in several biomedical areas. ${ }^{9}$ Liposomes improve medical therapies in a range of applications by 
stabilizing bioactive compounds, overcoming obstructions to cellular and tissue uptake, or improving biodistribution of substances to target sites in vivo. ${ }^{\mathbf{1 0 , 1 1}}$ Due to its encapsulation capability, liposome shows good potential in decreasing vascular irritation of anticancer drugs ${ }^{\mathbf{1 2 - 1 4}}$ in particular.

In our study, the inflammatory responses and related signaling pathways were investigated to illustrate the mechanism of vascular irritation induced by VB. Furthermore, to decrease the vascular irritation of $\mathrm{VB}$, venenum bufonis liposome (VBL) was prepared by the thin-film dispersion method. The vascular irritation as well as anticancer activity of VBL were evaluated and compared with that of VB.

\section{Materials and methods}

\subsection{Reagents and kits}

DLS, the extractions of venenum bufomis (VB), Chinese blister beetle (CB), red ginseng (RG) and Astragalus membranaceus (AM) were provided by Buchang Pharma. (Heze, China). The crude drug of VB was purchased from Anhui Boshida Agricultural Science and Technology Co., Ltd (Anhui, China) and authenticated by Jinao Duan. The VB met the qualitative and quantitative stipulations of the 2010 Chinese Pharmacopoeia. Voucher specimens were deposited in the Herbarium of Nanjing University of Chinese Medicine, Nanjing, China. Acetonitrile and formic acid were HPLC-grade from Merck (Darmstadt, Germany) and deionized water was purified using an EPED super purification system (Eped, Nanjing, China). The reference compounds cinobufagin (110803-200605), resibufogenin (110718-201108) were purchased from the Chinese National Institute of Pharmaceutical and Biological Products (Beijing, China). Bufalin (MUST-13091302), bufotalin (130828), gamabufalin (130809), and arenobufagin (130801) were purchased from Beijing Putian Tongchuang Biotechnology Co. Ltd (Beijing, China).

Soy phosphatidylcholine (SPC), soy hydro-phosphatidylcholine (HSPC), and cholesterol (Chol) were purchased from AVT (Shanghai, China).

IL-1 $\beta$, IL-6, TNF- $\alpha$ and IL-10 were determined by ELISA kits produced by Nanjing Jiancheng Technology Co., Ltd. (Nanjing, China) by following the corresponding instructions. All antibodies used in the present study were purchased from Cell Signaling Technology (Danvers, USA). The details of primary antibodies are listed in ESI. $\dagger$ All other reagents were obtained from Sinopharm Chemical Reagent Co., Ltd. (Nanjing, China), unless otherwise stated.

HepG2 cell and A549 cell were purchased from Division of Life Science and Center for Chinese Medicine, The Hong Kong University of Science and Technology.

\subsection{The preparation of bufadienolides in VB and the chemical analysis}

VB powder was refluxed for $30 \mathrm{~min}$ with dichloromethane 3 times with a mass and volume ratio of $1: 10$. The extracts were combined, concentrated under reduced pressure, dried sufficiently and weighed finally.
HPLC analysis was performed using a Hypersil ODS C18 (250 $\mathrm{mm} \times 4.6 \mathrm{~mm}, 5 \mu \mathrm{m})$ column, and the temperature was maintained at $25{ }^{\circ} \mathrm{C}$. The mobile phase was composed of A (acetonitrile) and $\mathrm{B}(0.5 \%$ potassium dihydrogen phosphate aqueous solution, $\mathrm{pH} 3.2$ ) using a gradient elution of $65-58 \% \mathrm{~B}$ at $0-7.5 \mathrm{~min}, 58-52 \%$ $\mathrm{B}$ at $7.5-13 \mathrm{~min}, 52-35 \% \mathrm{~B}$ at $13-30 \mathrm{~min}$, and $35-65 \% \mathrm{~B}$ at $30-$

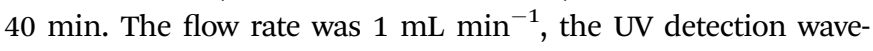
length was $296 \mathrm{~nm}$, and injection volume was $20 \mu \mathrm{L}$.

\subsection{The preparation of VBL}

The thin film hydration method was selected to prepare the crude liposome suspension. ${ }^{15}$ Briefly, lipid ingredients including SPC, HSPC, Chol and VB extract were dissolved in $\mathrm{CHCl}_{2}$ and dried on a rotary evaporation in a round-bottomed flask at $43{ }^{\circ} \mathrm{C}$. The lipid film was further dried under vacuum and hydrated with phosphate buffered saline (PBS, pH 7.4) at $50{ }^{\circ} \mathrm{C}$ for $15 \mathrm{~min}$. The lipid suspension was then homogenized at 1000 bsi for 9 cycles on a high pressure homogenizer to get the uniformity of the liposome particle size and reach the desired particle size range.

The average diameter, polydispersity index and zeta potential were measured using a Malvern Zetasizer 3000 system. All of the dynamic light scattering (DLS) measurements were performed at $25{ }^{\circ} \mathrm{C}$, at a scattering angle of $90^{\circ}$. The zeta potential values were calculated using the Smoluchowski equation. Transmission electron microscopy was used to estimate the morphology of the liposome. Filtration by a microspore film of $0.45 \mu \mathrm{m}$ was chosen for the determination of the entrapment efficiency of VBL.

\subsection{Ethics statement}

Animal welfare and experimental procedures were carried out in accordance with the guide for the care and use of laboratory animals (National Research Council, Washington, DC, USA) and the guidelines of the Animal Ethics Committee of Nanjing university of Chinese medicine. The experiment was approved by the institutional ethical committee Center for Drug Safety Evaluation and Research.

\subsection{Animals}

A total of nine rabbits (3 males and 6 females, SCXK (HU) 20140004) were acquired from Shanghai Laboratory Animal Co., Ltd (SLAC). (Shanghai). The experimental animals were allowed free access to water and mouse chow and were housed under controlled environmental conditions (constant temperature, humidity, and $12 \mathrm{~h}$ dark/light cycle). Rabbits were acclimated to the environment for 7 days before the experiment.

\subsection{Experimental protocol}

According to our previous studies, the dosage of VB was $0.12 \mathrm{mg}$ $\mathrm{kg}^{-1}$ to rabbits. In this study, animals were divided into three groups: (1) the control group (K); (2) the VB group (VB); (3) the VBL group (VBL). VB or VBL were injected into the veins of the rabbit ears, and the duration of injection was 3-4 minutes. 
At $6 \mathrm{~h}$ after the administration of VB or VBL, the rabbits were subjected to cardiac blood collection and sacrificed. The blood samples were centrifuged at $5000 \mathrm{rpm}$ for $15 \mathrm{~min}$. The supernatant was collected and set aside at $-80{ }^{\circ} \mathrm{C}$ for analysis of the inflammatory cytokines. After the sacrifice of the rabbits, the ear samples were divided into three parts: one part for the histopathological observation, another for the immunohistochemistry analysis, and the third part for the western blot analysis.

\subsection{The rabbit ear vein irritation test for $\mathrm{VB}$ and VBL}

Macroscopic observation and pathological examination were used to assess the vascular irritation. For macroscopic observation, the notations used were as follows: symptoms 0 for no obvious irritation; 1 for erythema; 2 for edema; 3 for ulcers; severity: + mild; ++ obvious; +++ severe.

For pathological examination, the ears were fixed in buffered neutral $10 \%$ formalin, embedded in paraffin and sectioned at 5 $\mu \mathrm{m}$ for staining with haematoxylin and eosin and visualized under light microscopy (Nikon, Tokyo, Japan) at $100 \times$ magnifications. According to the scope and severity of the result on the ear, it was graded on a scale of $0-3: 0=$ none, $1=$ mild, $2=$ moderate, 3 = severe.

\subsection{Determination of inflammatory cytokines}

The serum levels of IL-1 $\beta$, IL-6, TNF- $\alpha$ and IL-10 were determined using an enzyme-linked immunosorbent assay (ELISA) kit (Jiancheng Bioengineering Inc., China) in our study, according to the manufacturer's instructions.

\subsection{Western blot analysis}

The samples were homogenized in ice-cold RIPA buffer $(0.1 \%$ phenylmethylsulfonyl fluoride). The solution was centrifuged and the supernatant was collected. The protein concentration was determined using a BCA protein assay kit. Protein extracts were loaded using SDS-polyacrylamide gel electrophoresis. The PVDF membranes with transferred proteins were blocked with $5 \%$ skimmed milk for two hours. The membranes were incubated with primary antibodies of TLR2, TLR4, MyD88, p-Erk,

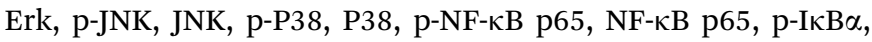
$\mathrm{I} \kappa \mathrm{B} \alpha$ and $\beta$-actin (CST, Danvers, USA) and $\beta$-actin (inner control) at $4{ }^{\circ} \mathrm{C}$ overnight. After the membranes were incubated with a secondary antibody, the bands were visualized by chemiluminescence detection reagents and a gel imaging system. Densitometric measurements were obtained using Image J software (National Institutes of Health, USA).

\subsection{Immunohistochemistry}

The expression of TLR2, TLR4 and p-NF- $\kappa \mathrm{B}$ p65 was further examined by immunohistochemistry staining. The procedure was performed as follows: the paraffin sections from different samples were deparaffinized, rehydrated and incubated in $3 \%$ hydrogen peroxide. Then, the samples were blocked with $3 \%$ BSA and incubated with primary antibody at $4{ }^{\circ} \mathrm{C}$ overnight. On the next day, the secondary antibody and three antibodies were treated for 20 min at $37{ }^{\circ} \mathrm{C}$. Samples were stained with DAB, restrained with hematoxylin, and observed under a microscope.

\subsection{In vitro anti-tumor activity test}

The in vitro anti-tumor activity of VB and VBL was determined using an MTT assay. Basically, human hepatocellular liver carcinoma cell line (HepG2) and adenocarcinomic human alveolar basal epithelial cells (A549) were transferred to 96-well tissue culture plates at $3 \times 10^{3}$ cells and $5 \times 10^{3}$ cells per well $12 \mathrm{~h}$ prior to drug treatment, respectively. The culture medium was then replaced with $90 \mu \mathrm{L}$ of medium and $10 \mu \mathrm{L}$ serial dilutions of VB dissolved in dimethylsulfoxide (DMSO, the final concentration in medium was less than $0.1 \%$ ) or VBL. Following $12 \mathrm{~h}$ incubation at $37{ }^{\circ} \mathrm{C}$ with $5 \% \mathrm{CO}_{2}$, the above operation was repeated and incubated at $37{ }^{\circ} \mathrm{C}$ with $5 \% \mathrm{CO}_{2}$ for another $12 \mathrm{~h}$. Then, the medium was removed and a total of $100 \mu \mathrm{L}$ MTT stock solution $\left(5 \mathrm{mg} \mathrm{mL}^{-1}\right.$ ) was added to each well, and the plates were incubated for $4 \mathrm{~h}$ at $37{ }^{\circ} \mathrm{C}$ with $5 \% \mathrm{CO}_{2}$. The solution was removed and $150 \mu \mathrm{L}$ DMSO was added to dissolve the blue formazan crystals converted from MTT. Cell viability was assessed by the absorbance at $570 \mathrm{~nm}$ measured on a Biorad microplate reader.

\subsection{In vivo anti-tumor activity test}

The tumor model was established as followed: ascitic $\mathrm{H}_{22}$ cells of murine carcinoma were maintained in our laboratory. The mice bearing the ascetic-type hepatoma were sacrificed following abdominal injection of the $\mathrm{H}_{22}$ cells for seven days. The skin was sterilized and removed, and the ascitic fluid was drawn through the muscles of the abdominal wall using a sterile syringe, which was diluted with normal saline to $10 \times$ $10^{6}$ cell solution. For solid tumor growth, $0.2 \mathrm{~mL}$ of diluted solution was injected subcutaneously at the right axilla of mice. ${ }^{16}$

When the tumors reached palpable sizes, the mice were randomized into the following groups (10 mice per group): control group injected daily with saline via intravenous injections; positive control group received $5-\mathrm{Fu}\left(10 \mathrm{mg} \mathrm{kg}^{-1}\right)$ via intravenous injections everyday; three VB groups with different dosages (VB-L group $1.6 \mathrm{mg} \mathrm{kg}{ }^{-1}$, VB-M group $3.2 \mathrm{mg} \mathrm{kg}^{-1}$, VB$\mathrm{H}$ group $4.8 \mathrm{mg} \mathrm{kg}^{-1}$ ), which received VB solution daily through intravenous injections; VBL group $\left(1.2 \mathrm{mg} \mathrm{kg} \mathrm{kg}^{-1}\right)$ through intravenous injection daily.

Throughout the experiments, the mice were weighed every 4 days. During the experiment period, side effects including weight loss, change in behavior and dietary, response to stimulation, ruffling of fur and psychosis (distress) were monitored. When a mouse died or met an early removal criterion and sacrificed, the number of days of survival was recorded. Inhibition of tumor was calculated using the formula: inhibition of tumor $(\%)=(1-$ average tumor weight in treated group/average tumor weight in control) $\times 100$.

\subsection{Statistical analysis}

Data were normally distributed and presented as mean \pm S.E.M. Results were analyzed by the analysis of variance (ANOVA) with 
Tukey's post hoc test using SPSS 17.0 (SPSS Inc., USA). $P$ value < 0.05 was considered to be significant.

\section{Results}

\subsection{The characterization of bufadienolides in VB}

Bufadienolides such as cinobufagin, resibufogenin, bufalin, bufotalin, gamabufalin and arenobufagin are the main components in the VB extract. The total mass of the six major bufadienolides is $15.07 \%$ of VB. Cinobufagin, resibufogenin, bufalin, bufotalin, gamabufalin and arenobufagin accounted for $5.73 \%, 2.84 \%, 2.06 \%, 1.77 \%, 0.87 \%$, and $1.80 \%$ of $\mathrm{VB}$, respectively. Cinobufagin and resibufogenin, the marker components for the quality control of VB, account for $8.57 \%$ of VB, and $56.88 \%$ of the six bufadienolides. The representative chromatogram of VB is shown in Fig. 1.

\subsection{The preparation of VBL}

We successfully prepared the composite phospholipid liposome of VB with the following characterizations: the mean size VBL was $99.51 \pm 4.23 \mathrm{~nm}$ with the zeta potential $-2.68 \pm 0.29 \mathrm{mV}$, and the liposome seemed to be of spherical or ellipsoidal shape. The particle size distribution and the transmission electron micrographs of the VBL are shown in Fig. 2. The encapsulation efficiency (EE) values were all $>80 \%$.

\subsection{Rabbit ear vein irritation test of VB and VBL}

Macroscopic observation showed that after single injection of VB and VBL to rabbits, both the ear vein in the two groups showed different degrees of erythema. Overall, VBL group showed less irritation than the VB group. No irritation symptom was observed in the rabbits in the control group (Table 1).

Pathological examination showed that irritation of VBL was less severe than that of the VB group. The main pathological features were swelling of the vascular endothelium swelling, vascular edema, bleed and inflammatory cell infiltration. No pathological change was found in the control group (Fig. 3, Table 2).

Considering the macroscopic observation and the histopathological results: VB and VBL exhibited different degrees of irritation on the rabbit ear vein, and VB induced vascular irritation was alleviated after liposome encapsulation.

\subsection{Effects of VB and VBL on inflammatory cytokines}

The levels of serum pro-inflammatory cytokines IL-1 $\beta$, IL-6 and TNF- $\alpha$ exhibited a notable augment in the VB group, while antiinflammatory cytokine IL-10 exhibited a decreasing trend, compared with control group $(P<0.01)$. Liposome encapsulation could significantly reverse the elevations of these proinflammatory cytokines induced by VB (Fig. 4). These results suggested that the release of VB induced pro-inflammatory cytokines can be attenuated after liposome encapsulation.

\subsection{Effect of VB and VBL on histological changes}

The histological changes of the vein in rabbit ears between the normal control, VB and VBL groups are depicted in Fig. 5. These results suggested that VB induced vascular irritation was attenuated after liposome encapsulation.
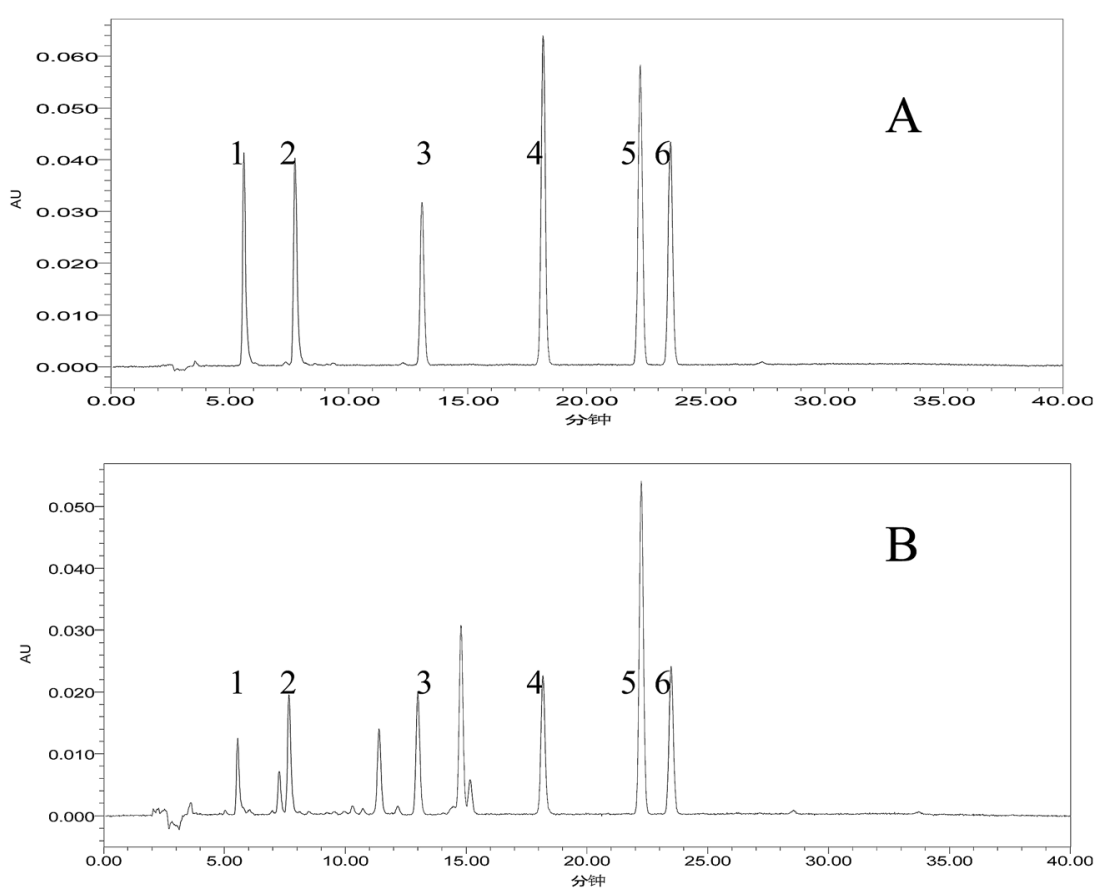

Fig. 1 The chromatogram of mixed standard compounds and VB extract analyzed by HPLC. (A) Six standard compounds: (1) gamabufalin; (2) arenobufagin; (3) bufotalin; (4) bufalin; (5) cinobufagin; (6) resibufogenin. (B) The chromatogram of VB extract. 

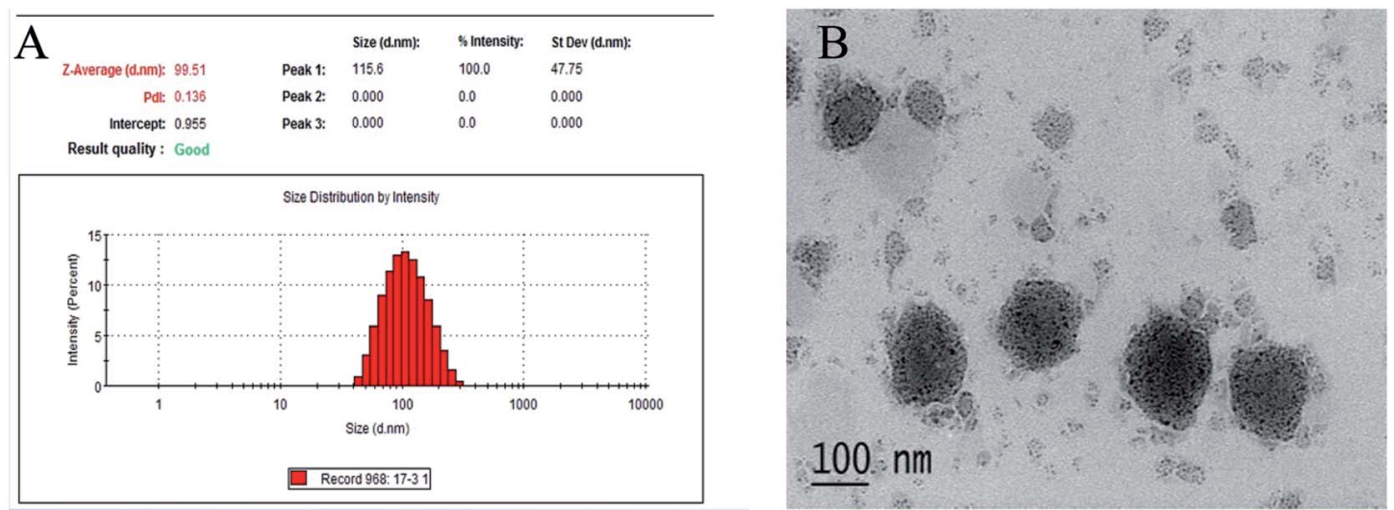

Fig. 2 The size distribution (A) and transmission electron micrographs of VBL (B).

Table 1 Visual evaluation after VB and VBL administration

\begin{tabular}{|c|c|c|c|c|c|}
\hline Groups & Number & Weight (kg) & Sex & Before & After \\
\hline \multirow[t]{3}{*}{ Control } & 1 & 2.2 & 0 & 0 & 0 \\
\hline & 2 & 2.3 & q & 0 & 0 \\
\hline & 3 & 2.3 & q & 0 & 0 \\
\hline \multirow[t]{3}{*}{ VB } & 1 & 2.1 & o & 0 & $1(++)$ \\
\hline & 2 & 2.5 & q & 0 & $1(++)$ \\
\hline & 3 & 2.0 & $q$ & 0 & $1(++)$ \\
\hline \multirow[t]{3}{*}{ VBL } & 1 & 2.2 & 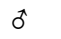 & 0 & $1(++)$ \\
\hline & 2 & 2.0 & q & 0 & $1(++)$ \\
\hline & 3 & 2.1 & 우 & 0 & $1(+)$ \\
\hline
\end{tabular}

\subsection{Effects of VB and VBL on TLR/MAPK/NF- $\kappa B$ signaling}

Next, we examined the related signaling pathway underling the VB-induced inflammation. Our results demonstrated that the increased expression of TLR2, TLR4, and MyD88, p-Erk, p-JNK,

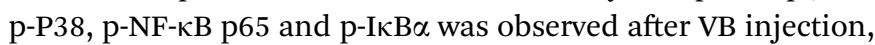
compared with control group. On the contrary, decreased expression of TLR2, TLR4, and MyD88, p-Erk, p-JNK, p-P38, p$\mathrm{NF}-\kappa \mathrm{B}$ p65 and $\mathrm{p}-\mathrm{I} \kappa \mathrm{B} \alpha$ was observed in the VBL group, compared with VB group (Fig. 6).

Immunohistochemistry analysis suggested the up-regulation of TLR2, TLR4 and p-NF- $\mathrm{B}$ p65 in the VB group, compared with the control group. VBL administration decreased VB induced overexpression of TLR2, TLR4 and $\mathrm{p}-\mathrm{NF}-\kappa \mathrm{B}$ p65. These results
Table 2 Pathological evaluation after VB and VBL administration

\begin{tabular}{lllll}
\hline Groups & Number & Edema & Bleeding & Inflammatory cell infiltration \\
\hline \multirow{2}{*}{ Control } & 1 & 0 & 0 & 0 \\
& 2 & 0 & 0 & 0 \\
VB & 3 & 0 & 0 & 0 \\
& 1 & 2 & 1 & 2 \\
& 2 & 2 & 1 & 3 \\
VBL & 3 & 2 & 1 & 3 \\
& 1 & 1 & 1 & 1 \\
& 2 & 2 & 0 & 2 \\
& 3 & 1 & 1 & 1
\end{tabular}

suggested that VB-induced vascular irritation through mediating TLR/MAPK/NF- $\mathrm{B}$ signaling pathway can be attenuated after liposome encapsulation.

\subsection{In vitro anti-tumor activity of $\mathrm{VB}$ and $\mathrm{VBL}$}

Proliferation of HepG-2 and A549 cells was inhibited by both VB and VBL in a concentration-dependent manner. $\mathrm{IC}_{50}$ of $\mathrm{VB}$ and VBL for HepG-2 cell were $1.511 \mu \mathrm{g} \mathrm{mL}{ }^{-1}$ and $0.579 \mu \mathrm{g} \mathrm{mL}^{-1}$, respectively; $\mathrm{IC}_{50}$ of $\mathrm{VB}$ and $\mathrm{VBL}$ for A549 cell was $5.744 \mu \mathrm{g} \mathrm{mL}^{-1}$ and $1.430 \mu \mathrm{g} \mathrm{mL} \mathrm{m}^{-1}$, respectively. These data showed that the cytotoxicity of liposomes encapsulated with VB was better than that of VB in both cells. In addition, the cytotoxicity of VB and

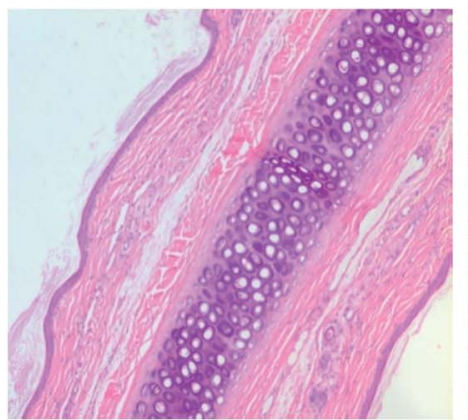

Control

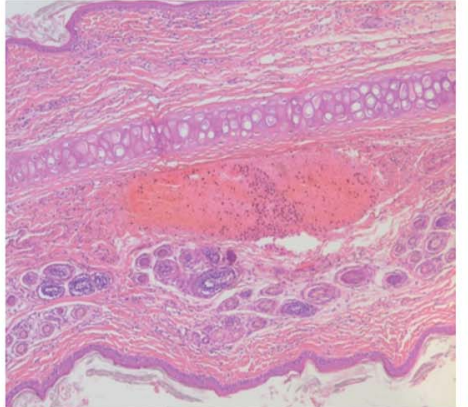

VB

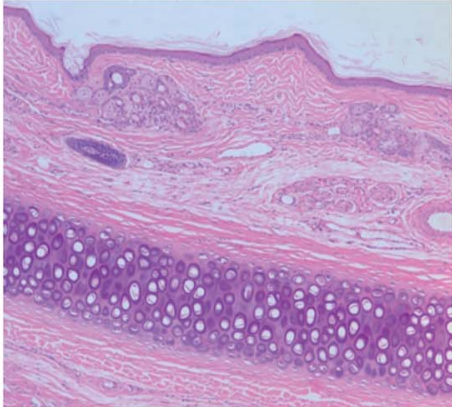

VBL

Fig. 3 Histopathological examination after VB and VBL administration $(H E \times 100)$. 

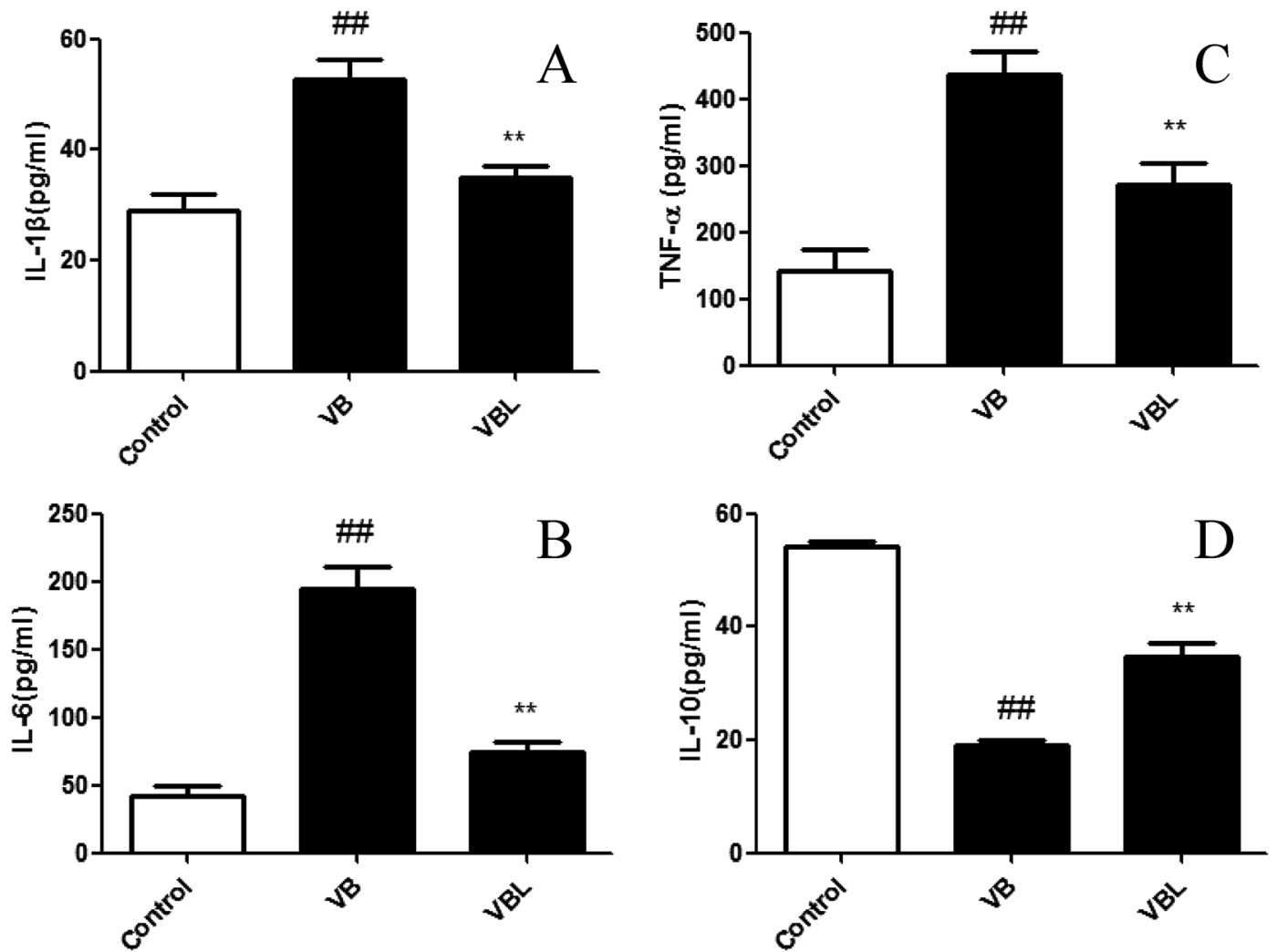

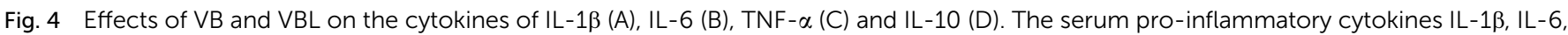
TNF- $\alpha$ and IL-10 from different groups were analyzed by ELISA kits. All values given are the mean \pm SD. \#\#p<0.01 vs. control group ( $n=3$ ), and $* * p<0.01$ vs. VB group $(n=3)$.

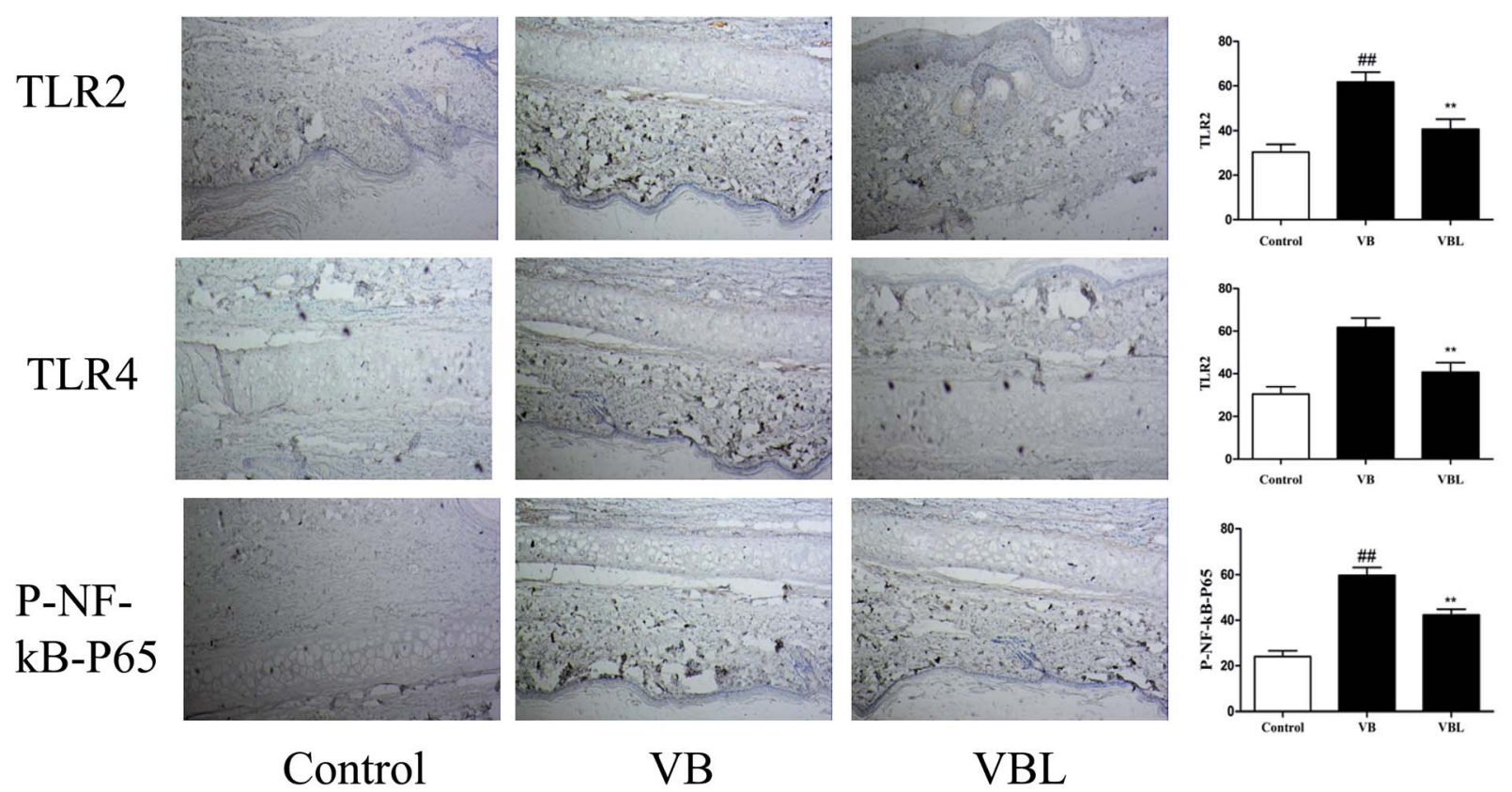

Fig. 5 The effect of VB and VBL on the TLR/NF- $\kappa B$ pathway studied by immunohistochemistry analysis. The expression of TLR2, TLR4 and $p-N F-$ $\kappa B$ p65 was investigated by immunohistochemical analysis.

VBL was concentration-dependent, as shown in Fig. 7. No cytotoxicity of VB unloaded liposome was observed in either cell-line.

\subsection{In vivo anti-tumor activity of $\mathrm{VB}$ and $\mathrm{VBL}$}

The treatment began the day after the mice were transplanted with tumor cells. As Fig. 8 shows, inhibitive rate of tumor 

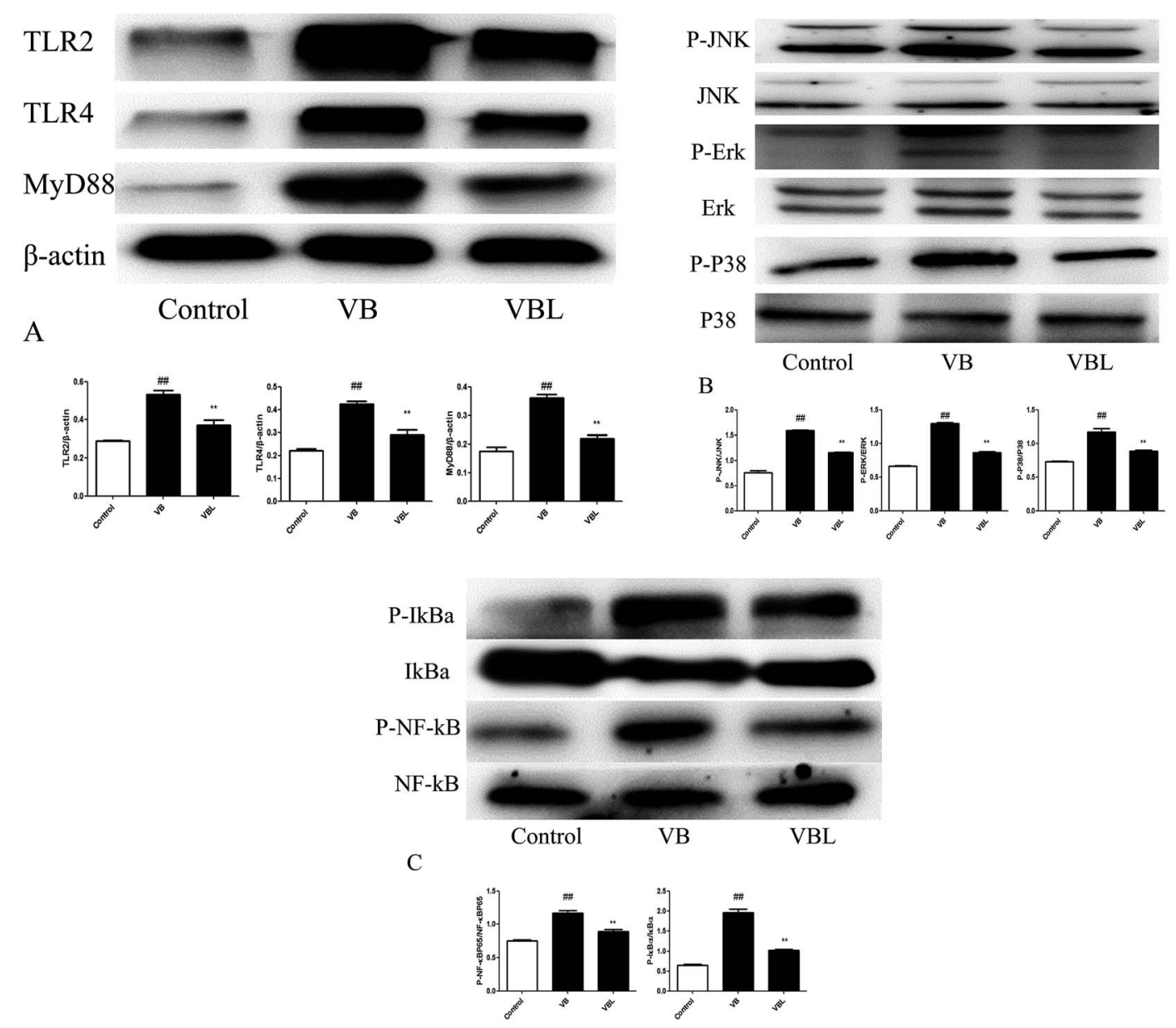

Fig. 6 The effect of $\mathrm{VB}$ and $\mathrm{VBL}$ on the TLR/NF- $\kappa B$ pathway $(\mathrm{A}-\mathrm{C})$. The expressions of related proteins in the TLR/NF- $\kappa B$ pathway were studied by western blot analysis in rabbits. The quantification was performed based on the data of three separated experiments. All values given are the mean \pm SD. \#\#p 0.01 vs. control group $(n=3)$, and $* * p<0.01$ vs. VB group $(n=3)$.

growth in VBL group was similar to that in the VB high dosage group $(p<0.05)$. At 11 days post-treatment, the mice in the control, VBL, and VB groups were all alive. There were no significant abnormalities in psychosis, status of activity, reaction to stimulation, appetite or depilation of mice in VB, control or VBL group. However, compared with VBL and VB groups, the mice in 5-Fu group lost more weight, as shown in Table 3. The results showed that VB still had antitumor activity after liposome encapsulation, and no side effects such as weight loss were observed in both VBL and VB groups.

\section{Discussion}

Anticancer drugs are generally administrated intravenously, often resulting in induced infusion phlebitis, which is the most common side effect of intravenous therapy. ${ }^{17,18}$ Some methods such as a change of formulation or pharmaceutical intervention $^{19,20}$ can alleviate the vascular irritation symptoms but the problem is not fundamentally solved. As reported previously, toxicities of brucine solution, BSL-SPC (brucine stealth liposomalsoy phosphatidylcholine), BSL-DPPC (brucine stealth liposomaldipalmitoyl phosphatidylcholine), BSL-HSPC (brucine stealth liposomal-hydrogenated soy phosphatidylcholine), and BSL-DSPC (brucine stealth liposomal-distearoyl phosphatidylcholine) were evaluated and compared; their $\mathrm{LD}_{50}$ values were $13.17,37.30$, $37.69,51.18$, and $52.86 \mathrm{mg} \mathrm{kg}^{-1}$, respectively, which proved that liposome encapsulation alleviated the toxicity of brucine. ${ }^{21}$ Moreover, pathological results showed that melittin liposomes modified with poloxamer 188 exhibit enhanced bioavailability, effective anticancer activity, reduced vascular irritation caused by MLT. ${ }^{13}$ After administration of CAP (capsaicin) or CAPLIP (liposomes with capsaicin), the histological analysis of wistar rats in the CAP group showed intense inflammation and ulcer formation in almost all bladder layers. Conversely, the CAPLIP group showed a smooth inflammatory reaction and hyperemia; liposome encapsulation alleviated the toxicity of capsaicin and effectively protected the bladder against the irritative action of the drug. ${ }^{12}$ Similar vascular irritation symptoms were also found in Chinese medicine injections containing VB such as DLS, ${ }^{22,23}$ and the incidence of the vascular irritation of DLS limits its treatment effect and continuation therapy. The pathogenesis of VB-induced vascular irritation is not fully understood, and thus a systematic study to reveal related mechanisms is of great practical significance. 

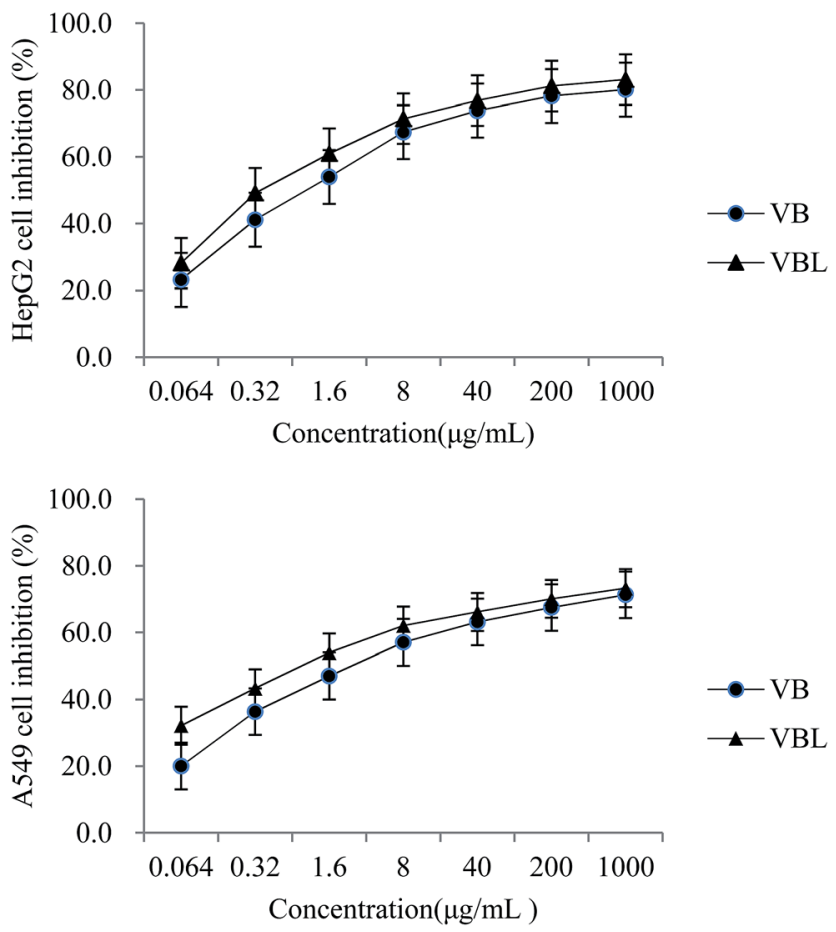

Fig. 7 Cytotoxicity of VB and VBL.

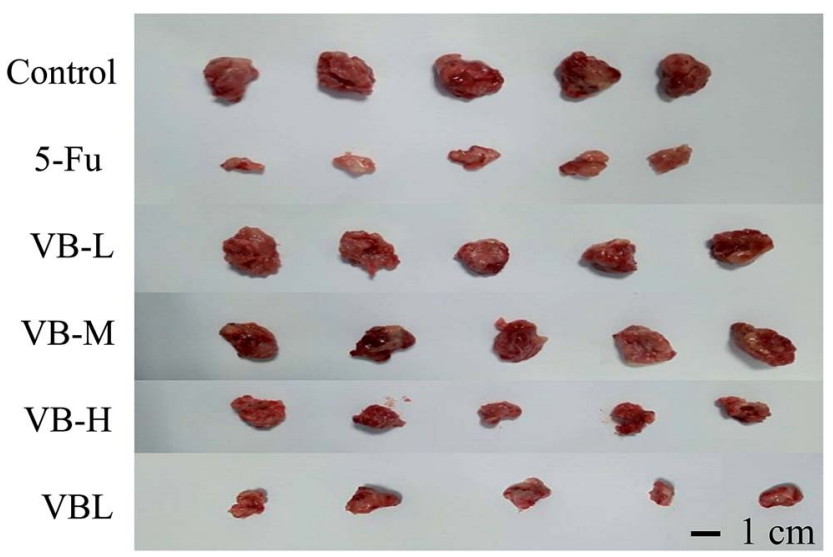

Fig. 8 Anti-tumor activity test in vivo.

Table 3 Effect of $V B$ and $V B L$ on the $\mathrm{H}_{22}$ mice

\begin{tabular}{|c|c|c|c|c|c|}
\hline \multirow[b]{2}{*}{ Groups } & \multicolumn{2}{|c|}{ Number of mice } & \multicolumn{2}{|c|}{ Body weight (g) } & \multirow{2}{*}{$\begin{array}{l}\text { Inhibition } \\
(\%)\end{array}$} \\
\hline & Beginning & End & Beginning & End & \\
\hline Control & 10 & 10 & $20.88 \pm 1.48$ & $21.60 \pm 1.32$ & 0 \\
\hline VB-L & 10 & 10 & $21.21 \pm 1.27$ & $22.29 \pm 1.13$ & 33.48 \\
\hline VB-M & 10 & 10 & $20.91 \pm 1.24$ & $21.82 \pm 1.13$ & 46.95 \\
\hline VB-H & 10 & 10 & $20.52 \pm 1.30$ & $22.03 \pm 1.16$ & 62.31 \\
\hline VBL & 10 & 10 & $20.73 \pm 1.90$ & $22.55 \pm 1.70$ & 61.90 \\
\hline 5-Fu & 10 & 10 & $20.51 \pm 1.18$ & $19.61 \pm 1.05$ & 68.57 \\
\hline
\end{tabular}

Liposomes exhibit diverse characteristics, such as the improved biocompatibility, enhanced capacity for self-assembly, capability to carry large drug payloads, and more importantly, potential physicochemical and biophysical properties which can regulate or control the drugs' biological characteristics. ${ }^{24,25}$ Liposomes are traditionally considered to be pharmacologically inactive with minimal toxicity because of the composition of natural phospholipids. ${ }^{\mathbf{1 1 2 6}}$ Composite phospholipids, with characteristics such as enhancing the activity, improving the entrapment efficiency and increasing the stability have been applied more and more in recent years. ${ }^{27-29}$ Based on the above characteristics of liposome encapsulation, reasons why liposomes can improve or maintain the activity of the drug can be explained from the following reasons. One is the natural targeting of the liposome, which can improve drug efficacy while decreasing distribution to non-target site. Low distribution of drugs at the non-target site will reduce the toxicity of drugs in non-target tissues, and natural phospholipids with good biocompatibility of the liposome composition also explains why liposomes are traditionally considered to have minimal toxicity. The reason why liposomes can reduce the vascular irritation is that encapsulation of drugs can avoid its direct contact with blood vessels. Compared with other traditional Chinese medicine injections, the release procedure from the liposome can avoid the rapid increased drug concentration in blood, which could be another factor leading to vascular irritation. In our study, composite phospholipids composed of SPC and HSPC ratios were optimized to prepare VBL, and bufadienolides in VB was further encapsulated into these lipids according to the best optimized composition.

After injecting VB into rabbit ears, the inflammatory process is initiated. The activated inflammatory cytokines then promote the expression of inflammatory mediators, which might damage the lysosomal membranes, leading to the release of lysosomal enzymes, free radicals, and pro-inflammatory cytokines, which further intensify the inflammatory responses. The inflammatory stress was implicated in the VB group by evident elevation of IL-1 $\beta$, TNF- $\alpha$ and IL-6. Liposomes encapsulation significantly attenuated VB-induced IL-1 $\beta$, TNF- $\alpha$ and IL-6 elevation in serum, which indicated that liposome encapsulation could attenuate VB induced inflammatory reaction.

TLRs belong to a super-family of pattern recognition receptors, and TLRs signaling is the host's important detector of foreign pathogens that controls the initial type-1 IFN reactions in the innate immune responses. ${ }^{30} \mathrm{~A}$ diverse range of TLR subtypes, such as TLR2 and TLR4, has the abilities to detect inflammatory stimulus and initiate an innate immune response against the inflammatory damages. ${ }^{31,32}$ Myd88 is a primary adaptor for almost all TLR signaling, besides the activation of TLR3 and Myd88-independent TLR4. Inflammation is an intricate progression with the activation of multiple signaling cascades. Upon activation, TLR induces the stimulation of interferon regulatory factors (IRFs) and NF- $\kappa \mathrm{B}$ signaling, which subsequently drives the production of pro-inflammatory cytokines. In the present study, VB injection significantly increased the levels of TLR2, TLR4, MyD88, and VB liposome treatment reversed the elevation.

Upon activation, TLR2 and TLR4 interact with MyD88 and initiate intricate cascades, including the activation mitogenactivated protein kinases (MAPKs) signaling. The p38 mitogen activated MAPK family is one of the most well investigated 
intracellular signal transduction cascades that monitor the maturation of proinflammatory cytokines. In VB group, the expression of p-Erk, p-JNK, and p-P38 was remarkable increased. After liposome encapsulation, the overexpression of these proteins induced by VB was significantly decreased. The maturation and production of pro-inflammatory cytokines is primary mediated by the NF- $\kappa \mathrm{B}$ cascades. ${ }^{33}$ In quiescent cells, the NF- $\kappa \mathrm{B}$ protein is inert when it interacts with the inhibitory kappa B (I $\mathrm{B}$ ) family in the cytoplasm. When stimulus is detected, I $\kappa \mathrm{B} \alpha$ is degraded by the activated I $\mathrm{B}$ kinase complex, which leads NF- $\kappa \mathrm{B}$ to translocate into the nucleus and interact with the target genes. ${ }^{34}$ The processed NF- $\kappa \mathrm{B}$ up-regulates the gene expression of pro-inflammatory cytokines, biological substrates, and chemokines enzymes etc., which ultimately leads to the inflammatory damages..$^{35,36}$ In our present study, the results indicated that $\mathrm{VB}$ induced over-expression of $\mathrm{p}-\mathrm{I} \kappa \mathrm{B} \alpha$, while VBL treatment abrogated the I $\kappa \mathrm{B} \alpha$ phosphorylation and degradation. At the same time, VB-induced nuclear translocation of NF- $\kappa$ B p65 was also after liposome encapsulation.

In summary, our study has demonstrated that VB induced vascular irritation, and increased serum levels of proinflammatory cytokines was related with the TLR/MAPK/NF- $\kappa$ B pathway. Liposome encapsulation of VB significantly attenuated VB induced pathological changes and inflammation. Moreover, the in vivo and in vitro anticancer activity of VB was also maintained after liposome encapsulation.

\section{Conflict of interest statement}

There is no conflict of interest among the authors.

\section{Abbreviations}

$\begin{array}{ll}\text { DLS } & \text { Delisheng } \\ \text { VB } & \text { Venenum bufonis } \\ \text { CB } & \text { Chinese blister beetle } \\ \text { RG } & \text { Red ginseng } \\ \text { AM } & \text { Astragalus membranaceus } \\ \text { VBL } & \text { Venenum bufonis liposome } \\ \text { IL-1 } \beta & \text { Interleukin-1 } \beta \\ \text { IL-6 } & \text { Interleukin-6 } \\ \text { TNF- } \alpha & \text { Tumor necrosis factor- } \alpha \\ \text { IL-10 } & \text { Interleukin-10 } \\ \text { TLR2 } & \text { Toll-like receptor 2 } \\ \text { TLR4 } & \text { Toll-like receptor } 4 \\ \text { MyD88 } & \text { Myeloid differentiation factor } 88 \\ \text { p-Erk } & \text { Phosphorylation extracellular regulated protein } \\ & \text { kinases } \\ \text { Erk } & \text { Extracellular regulated protein kinases } \\ \text { p-JNK } & \text { Phosphorylation c-Jun N-terminal kinase } \\ \text { JNK } & \text { c-Jun N-terminal kinase } \\ \text { p-P38 } & \text { Phosphorylation mitogen-activated protein kinase } \\ & \text { P38 } \\ \text { P38 } & \text { Mitogen-activated protein kinase P38 } \\ \text { p-NF- } \kappa B & \text { Phosphorylation nuclear factor } \kappa \text { B p65 } \\ \text { p65 } & \\ \text { NF- } \kappa B \text { p65 } & \text { Nuclear factor } \kappa \text { B p65 } \\ & \end{array}$

p-IкB $\alpha \quad$ Phosphorylation inhibitor of $\kappa \mathrm{B}$ alpha

$\mathrm{I} \kappa \mathrm{B} \alpha \quad$ Inhibitor of $\kappa \mathrm{B}$ alpha

MAPK Mitogen-activated protein kinase

NF- $\kappa$ B Nuclear factor $\kappa B$

SPC Soy phospholipids

HSPC Hydrogenated soy phospholipids,

Chol Cholesterol

EE Encapsulation efficiency

PBS Phosphate buffered saline

ELISA Enzyme-linked immunosorbent assay

BCA Bicinchoninic acid

HepG2 Hepatocellular liver carcinoma cell line

A549 Alveolar basal epithelial cells

DMSO Dimethyl sulfoxide

MTT 3-(4,5-Dimethylthiazol-2-yl)-2,5-diphenyltetr azolium bromide

$\mathrm{H}_{22} \quad$ Hepatoma 22

$\mathrm{IC}_{50} \quad$ Half maximal inhibitory concentration

\section{Acknowledgements}

This study was supported by the National Natural Science Foundation of China (grant number 81673563) and a project funded by the Priority Academic Program Development of Jiangsu Higher Education Institutions (PAPD). Thanks Rui Liu for the early guidance of this study; Thanks Zhenhua Zhu for some part of the data analysis; Thanks Zhenhui $\mathrm{Wu}$ for the helpment in the animal experiment.

\section{References}

1 X. Y. Chen and L. Luo, J. Mod. Oncol., 2006, 14, 378-380.

2 J. Cui, K. J. Nan, T. Tian, Y. H. Guo, N. Zhao and L. Wang, World J. Gastroenterol., 2007, 13, 5432-5439.

3 C. X. Lu, K. J. Nan, Y. L. Nie, Y. N. Hai and M. Jiao, Mol. Biol. Rep., 2010, 37, 3407-3412.

4 S. H. Wang, Y. C. Wang, Y. L. Nie, Y. N. Hai, H. F. Sun, Z. L. Yuan and K. J. Nan, Indian J. Pharm. Sci., 2013, 75, 578-584.

5 Y. B. Liu, Z. G. Zhou, P. Zhang, G. L. Wang and R. H. Yang, Modern Oncology, 2006, 14, 571-572.

6 F. F. Kong, X. Q. Tan and L. J. Guo, China Med. Her., 2011, 8, 102.

7 W. Q. Zhou and H. T. Bi, Chinese Journal of Misdiagnosis, 2007, 7, 3940.

8 J. M. Zhang, Journal of Qilu Nursing, 2007, 13, 68.

9 W. T. Al-Jamal and K. Kostarelos, Acc. Chem. Res., 2011, 44, 1094-1104.

10 J. M. Metselaar and G. Storm, Expert Opin. Drug Delivery, 2005, 2, 465-476.

11 G. A. Koning and G. Storm, Drug discovery today, 2003, 8, 482-483.

12 L. M. Cirino, D. M. Vergne, P. F. Santana, E. D. Almeida, L. P. Costa, R. L. Albuquerque-Junior, I. B. Lima-Verde, F. F. Padilha and J. C. Cardoso, An. Acad. Bras. Cienc., 2016, 88, 1539-1547. 
13 J. L. Tian, X. Ke, Z. Chen, C. J. Wang, Y. Zhang and T. C. Zhong, Pharmazie, 2011, 66, 362-367.

14 D. Liu, J. Xing, F. Xiong, F. Yang and N. Gu, Drug Dev. Ind. Pharm., 2017, 1-9, DOI: 10.1080/03639045.2016.1275670.

15 E. Pupo, A. Padron, E. Santana, J. Sotolongo, D. Quintana, S. Duenas, C. Duarte, M. C. de la Rosa and E. Hardy, J. Controlled Release, 2005, 104, 379-396.

16 M. Zhou, H. Higo and Y. Cai, Phytother. Res., 2010, 24, 827833.

17 G. B. Lewis and J. F. Hecker, Br. J. Anaesth., 1985, 57, 220233.

18 D. G. Maki and M. Ringer, Ann. Intern. Med., 1991, 114, 845854.

19 K. Nagata, N. Egashira, T. Yamada, H. Watanabe, Y. Yamauchi and R. Oishi, Supportive care in cancer : official journal of the Multinational Association of Supportive Care in Cancer, 2012, vol. 20, pp. 951-955.

20 T. Yamada, N. Egashira, H. Watanabe, K. Nagata, T. Yano, T. Nonaka and R. Oishi, Supportive care in cancer: official journal of the Multinational Association of Supportive Care in Cancer, 2012, vol. 20, pp. 1549-1553.

21 J. Chen, G. J. Yan, R. R. Hu, Q. W. Gu, M. L. Chen, W. Gu, Z. P. Chen and B. C. Cai, Int. J. Nanomed., 2012, 7, 3567-3577.

22 Y. Xiao, Y. B. Hao and Y. M. Xie, China J. Chin. Mater. Med., 2012, 37, 2824-2826.
23 H. J. Dong, L. J. Zhang, X. F. Zhai, Y. X. Chen, Y. Q. Liu, X. H. Yuan and W. W. Huo, J. Chin. Integr. Med., 2007, 5, 585-587.

24 N. Monteiro, A. Martins, R. L. Reis and N. M. Neves, J. R. Soc., Interface, 2014, 11, 20140459.

25 S. Hua and S. Y. Wu, Front. Pharmacol., 2013, 4, 143.

26 B. S. Ding, T. Dziubla, V. V. Shuvaev, S. Muro and V. R. Muzykantov, Mol. Interventions, 2006, 6, 98-112.

27 J. Chen, C. Q. He, A. H. Lin, F. Xu, F. Wang, B. Zhao, X. Liu, Z. P. Chen and B. C. Cai, Drug Dev. Ind. Pharm., 2014, 40, 244-251.

28 C. Zeng, W. Jiang, M. Tan, J. Xing and C. He, Pharmacogn. Mag., 2016, 12, 313-318.

29 C. Zeng, W. Jiang, M. Tan, X. Yang, C. He, W. Huang and J. Xing, Eur. J. Pharm. Sci., 2016, 85, 123-131.

30 M. A. Armant and M. J. Fenton, Genome Biol., 2002, 3, 3011. 31 P. Cejkova, I. Nemeckova, J. Broz and M. Cerna, Biomed. Pap., 2016, 160, 76-83.

32 R. S. Sauer, D. Hackel, L. Morschel, H. Sahlbach, Y. Wang, S. A. Mousa, N. Roewer, A. Brack and H. L. Rittner, Mol. Pain, 2014, 10, 10.

33 X. Y. Deng, H. Y. Li, J. J. Chen, R. P. Li, R. Qu, Q. Fu and S. P. Ma, Behav. Brain Res., 2015, 291, 12-19.

34 M. Karin and M. Delhase, Semin. Immunol., 2000, 12, 85-98. 35 P. J. Barnes, Int. J. Biochem. Cell Biol., 1997, 29, 867-870.

36 D. Xueyang, M. Zhanqiang, M. Chunhua and H. Kun, OncoTargets Ther., 2016, 7, 78764-78772. 